\title{
Single Centre Experience on Decision Making for Mechanical Thrombectomy Based on Single-Phase CT Angiography by Including NCCT and Maximum Intensity Projection Images - A Comparison with Magnetic Reso- nance Imaging after Non-Contrast CT
}

\author{
Myeong Soo Kim, ' Gi Sung Kim² \\ Department of Neurosurgery, Dong Kang Medical Center, Ulsan, Korea \\ Department of Radiology, ${ }^{2}$ Dong Kang Medical Center, Ulsan, Korea
}

Objective : The purpose of this study was to suggest that computed tomography angiography (CTA) is valuable as the only preliminary examination for mechanical thrombectomy (MT). MT after single examination of CTA including noncontrast computed tomography (NCCT) and maximum intensity projection (MIP) improves door-to-puncture time as well as results in favorable outcomes.

Methods : A total of 157 patients who underwent MT at Dong Kang Medical Center from April 2015 to March 2019 were divided into two groups based on the examination performed prior to MT : CTA group who underwent CTA with NCCT and MIP, and NCCT+magnetic resonance image (MRi) group who underwent MRI including perfusion images after NCCT. In the two groups, time to CTA imaging or NCCT+MRi imaging after symptom onset, and time to arterial puncture and reperfusion were characterized as time-related outcomes. The evaluation of vascular recanalization after MT was defined as a modified thrombolysis in cerebral infarction (mTICl) scale. National Institutes of Health Stroke Scale (NIHSS) was assessed at the time of the visit to the emergency room and modified Rankin Scale (mRS) was assessed after 90 days.

Results : Typically, there were 34 patients in the CTA group and 33 patients in the NCCT+MRi group. A significantly shorter delay for door-to-puncture time was observed (mean, $86 \pm 22.1$ vs. $176 \pm 47.5$ minutes; $p<0.01$ ). Also, a significantly shorter door-to-imege time in the CTA group was observed (mean, $13 \pm 6.8$ vs. $93 \pm 30.8$ minutes; $p<0.01$ ). Moreover, a significantly shorter onset-to-puncture time was observed (mean, $195 \pm 128.0$ vs. $314 \pm 157.6$ minutes; $p<0.01)$. Reperfusion result of $\mathrm{mTICl} \geq 2 \mathrm{~b}$ was $100 \%$ (34/34) in the CTA group and $94 \%$ (31/33) in the NCCT+MRi group, and $\mathrm{mTICl} 3$ in $74 \%$ (25/34) in the CTA group and $73 \%$ (24/33) in the NCCT+MRi group. Favorable functional outcomes (mRS score $\leq 2$ at 90 days) were $68 \%(23 / 34)$ in the CTA group and 60\% (20/33) in the NCCT+MRi group.

Conclusion : A single-phase CTA including NCCT and MIP images was performed as a single preliminary examination, which led to a reduction in the time of the procedure and resulted in good results of prognosis. Consequently, it is concluded that this method is of sufficient value as the only preliminary examination for decision making.

Key Words : Stroke · Thrombectomy · Computed tomography angiography.

- Received : May 30, 2019 •Revised : July 23, 2019 •Accepted : September 1, 2019

- Address for reprints : Myeong Soo Kim

Department of Neurosurgery, Dong Kang Medical Center, 239 Taehwa-ro, Jung-gu, Ulsan 44455, Korea

Tel : +82-52-241-1114, Fax : +82-52-241-1180, E-mail : 1004@jbnu.ac.kr, ORCID : https://orcid.org/0000-0003-4542-2912

This is an Open Access article distributed under the terms of the Creative Commons Attribution Non-Commercial License (http://creativecommons.org/licenses/by-nc/4.0) which permits unrestricted non-commercial use, distribution, and reproduction in any medium, provided the original work is properly cited. 


\section{INTRODUCTION}

Early recanalization of occluded vessels in acute stroke patients improves clinical outcomes and reduces mortality ${ }^{2,3,16,36)}$. In patients with large vessel occlusion (LVO), the use of recombinant tissue plasminogen activator (r-tPA) only does not lead to high recanalization probability ${ }^{28)}$. In recent trials, a variety of instruments have been used and the high recanalization results have been confirmed in those trials. The development of instruments for mechanical thrombectomy (MT) has also permitted us to expect better recanalization ${ }^{18,37,46)}$. Numerous institutions are working on realizing early recanalization by attempting to reduce the lagging time to initiate the procedure after the patient is transferred to the emergency room (ER) and to improve the effective procedure for recanalization $^{1,25,30,45)}$.

Preliminary examinations are essential for decision making for endovascular treatment (EVT). Numerous studies confirmed the use of r-tPA after non-contrast Computed Tomography (NCCT) and performing EVT after confirmation of computed tomography angiography (CTA), CT perfusion (CTP), and magnetic resonance image (MRI) with perfusion images ${ }^{3,6,17,18,39)}$. In actual clinical practice, the procedure is performed after confirmation of CTA (or CTP) after NCCT, but there exists a tendency to decide on the procedure after MRI examination with perfusion images ${ }^{15,25,27)}$.

In CTA protocol, imaging, thin-section NCCT, and maximum intensity projection (MIP) images can identify the presence of hemorrhage, the exact thromboembolic occlusion site, and the structure of the extra-intracranial artery including the aortic arch for the endovascular procedure, and can be evaluated the pial collaterals for prediction of prognosis ${ }^{15,32)}$.

In this study, we investigated the clinical outcomes of patients who had undergone MT after a single-phase CTA imaging including NCCT and MIP images to reduce the lag time between the patient arrival and the initiation of the procedure. Compared with the group which underwent MRI after NCCT, our new attempt not only led to a reduction in the time to start the procedure but also led to good results in terms of prognosis. Therefore, we propose the importance of this method as the only preliminary examination for decision making.

\section{MATERIALS AND METHODS}

This retrospective study was approved by the Institutional Review Board (No. 2019-06-03).

\section{Patient selection}

A retrospective cohort study was performed by including patients who underwent MT for LVO performed at Dong Kang Medical Center. A total of 157 patients who underwent MT at our institution from April 2015 to March 2019 were divided into two groups based on the examination performed prior to MT : for all stroke patients who were eligible for inclusion criteria, some of them were allocated into a group (CTA) who had taken CTA with NCCT and MIP as a sole examination prior to procedure at the time of arrival on the ER and the others were allocated into a group (NCCT+MRi) who underwent MRI including perfusion images after NCCT. In the CTA group, CTA imaging with NCCT and MIP was performed for patients with all the neurological symptoms who transferred to the ER. Blood sampling for the decision of r-tPA administration was performed during intravenous (IV) line acquisition for imaging to collect rapid blood test results. After securing the IV line, the patient was immediately transferred to the imaging laboratory for imaging. During the CTA imaging, thin-section NCCT and CTA images were taken sequentially and were confirmed in real time. The prepared MIP images were confirmed within minutes for accurate diagnosis of lesion and decision making. The CTP was not performed (Fig. 1).

The inclusion criteria were as follows : patients who arrived within 4.5 hours after symptom onset, lacked hypodense lesion on CTA image who arrived within 8 hours even after 4.5 hours of symptoms onset, with thromboembolic occlusion of large vessel (internal carotid artery [ICA], middle cerebral artery [MCA], anterior cerebral artery [ACA], posterior cerebral artery $[\mathrm{PCA}]$, basilar artery $[\mathrm{BA}]$, vertebral artery $[\mathrm{VA}])$, and patients with National Institutes of Health Stroke Scale (NIHSS) 4 or higher.

Patients in the NCCT+MRi group underwent NCCT imaging immediately after they arrived at the ER, followed by MRI imaging with perfusion images. The inclusion criteria for MT applies a universal indication : patients with LVO, patients who arrived within 8 hours after symptom onset, NIHSS 4 or higher, patients without evidence of cerebral hemorrhage, pa- 
tients with a perfusion-diffusion mismatch in MRI with infarct core volume less than $1 / 3$, and patients with perfusion defect more than $2 / 3^{39)}$.

Patients with cerebral hemorrhage, patients without LVO, and patients with severe hypodensity on NCCT were excluded before MT through CTA examination. In addition, even if there was an LVO and if the physician could not proceed to decision making with CTA alone, MRI examination with perfusion images was performed and the patients who were not eligible were excluded from the study group. Patients who were transferred, who developed stroke during hospitalization, and who underwent other examination than CTA before the procedure were excluded. Of 157 patients, 49 patients performed NCCT+MRi as pre-examination of MT, 53 patients CTA, 30 patients CTA+MRI, four patients NCCT alone, one MRI alone, one took CT+CTA, nine tested at other hospitals, and 10 patients had in-hospital strokes. The 16 patients ex- cluded from the NCCT+MRi group were four patients with onset-to-door time (OTD) of more than eight hours, seven with unclear onset time (wake-up, un-witnessed daytime strokes), and five patients with less than 4 NIHSS score. The 19 patients excluded from the CTA group were 14 patients with OTD from eight hours to less than 12 hours, and five patients with less than 4 NIHSS score.

IV r-tPA was administered to eligible patients in both the groups following guidelines for the early management of acute ischemic stroke (AIS) ${ }^{13)}$.

\section{Image protocol and analysis}

\section{Single-phase CTA}

An Aquilion CT scanner (Toshiba Medical Systems, Tokyo, Japan) was used. After the acquisition of nonenhanced CT data, contrast-enhanced CTA was performed. CTA was per-
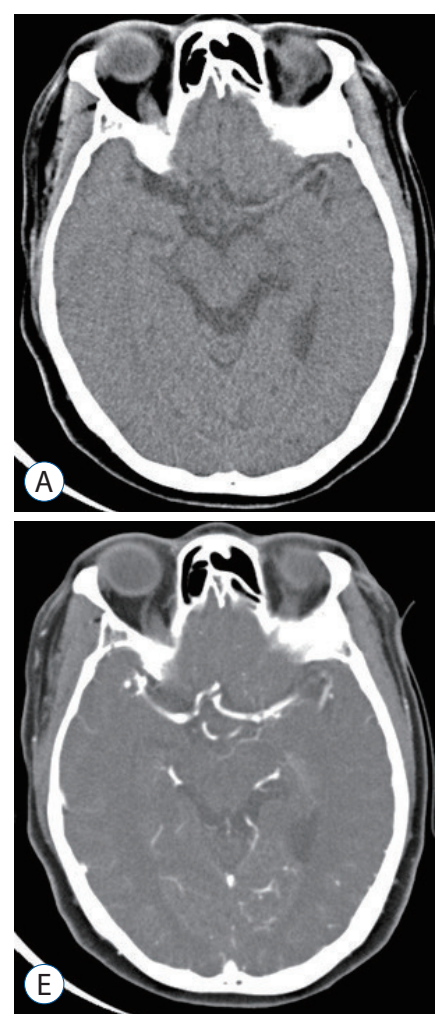
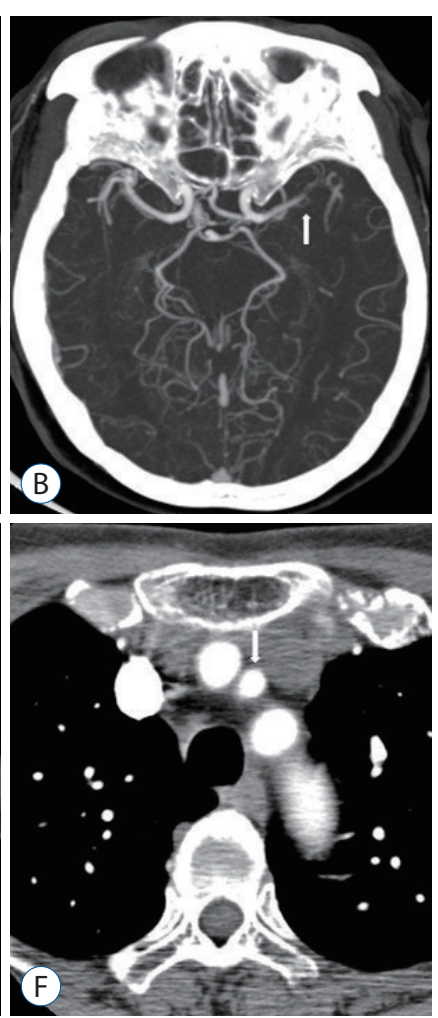
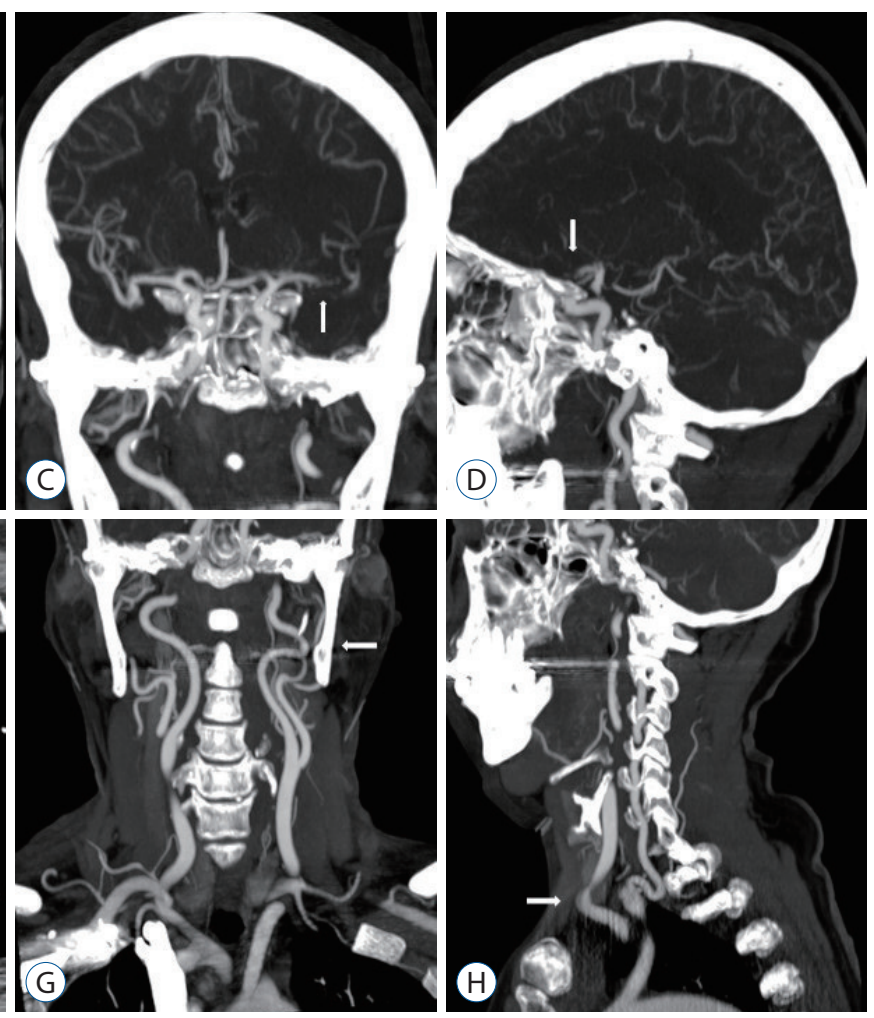

Fig. 1. Single-phase CT angiography images including NCCT and MIP images. A 62-year-old woman with a left M1 MCA occlusion. A : Thin-section NCCT (no hypodense lesion, no hemorrage). B : MIP intracranial axial image (arrow, left M1 MCA occlusion). C : MIP intracranial coronal image (arrow, left M1 MCA occlusion). D : MIP intracranial sagittal image (arrow, left M1 MCA occlusion). E : Contrast-enhanced CT image. F : Axial image showing the aortic arch (arrow, left CCA; origin of Brachiocephalic trunk, left CCA, and left subclavian artery). G : MIP extracranial coronal image (arrow, no stenosis of left CCA-ICA and tortuosity of left cervical ICA). H : MIP extracranial sagittal image (arrow, tortuosity of left mid CCA). CT : computed tomography, NCCT : non-contrast computed tomography, MIP : maximum intensity projection, MCA : middle cerebral artery, CCA : common carotid artery, ICA : internal carotid artery. 
formed by axial acquisition from the aortic arch to the vertex using a $100 \mathrm{kVP}, 250 \mathrm{~mA}, 0.5 \mathrm{~mm}$ detector collimation, 240 $\mathrm{mm}$ D-FOV with a rotation speed of $0.5 \mathrm{sec} /$ rotation. A total of $60 \mathrm{~mL}$ of contrast material (iopamidol, Pamiray 370; Dongkook Pharmaceuticals, Seoul, Korea) was administered intravenously using a power injector at $4.0 \mathrm{~mL} / \mathrm{sec}$ followed by 50 $\mathrm{mL}$ normal saline chase at a rate of $4.0 \mathrm{~mL} / \mathrm{sec}$. The axial contrast-enhanced images were reconstructed at 3-mm thickness with no intervals. Thick-section axial, coronal and sagittal MIP at 20-mm thickness and 3-mm intervals were also reconstructed. Reconstructed images of thin-section NCCT and CTA were taken from aortic arch to vertex, and at the same time, the images were transferred to a picture archiving and communication system (PACS). For identifying the intracranial lesion, MIP images were reconstructed from the skull base to the vertex in the order of axial, coronal, and sagittal. Extracranial MIP images were reconstructed from the aortic arch to the skull base in the order of coronal and sagittal images in order to identify common carotid artery (CCA), ICA, and VA. Subsequently, the images were uploaded to the PACS sequentially as soon as they were reconstructed so that they could be confirmed in real time.

\section{Non-contrast CT and MRI}

NCCT and MRI imaging were acquired by following the usual protocols. NCCT was performed with standard protocols using an Aquilion CT scanner (Toshiba Medical Systems). Axial NCCT images were obtained with $120 \mathrm{kV}, 300 \mathrm{~mA}$, and 5-mm-section-thickness reconstruction. MRI imaging with MR angiography and perfusion imaging were performed on either 1.5T or 3T scanners (Avanto 1.5T or Skyra 3T; Siemens, Erlangen, Germany) using standard departmental and vendor protocols.

Pial collateral circulation was defined on the CTA as good, moderate, or poor (using Menon pial arterial filling scale, score 5 and 4 : good, 3 and $2:$ moderate, below $1:$ poor ${ }^{32)}$ (Table 1, Figs. 2-4).

\section{Endovascular procedure}

Various instruments were used as per the characteristics of each patient, and the conventional procedure and technique were applied. The decision on MT was made at the discretion of the physician based on the patient's clinical status and the results of digital subtraction angiography (DSA). According to the MT protocol, in most cases, contact aspiration thrombectomy was performed. In case of failure of recanalization after several attempts, a stent retriever was used. However, a stent retriever was preferred instead of aspiration catheters in cases of difficult target access due to high vascular tortuosity or when it was not possible to insert the aspiration catheters due to vascular stenosis.

A shuttle guide sheath or balloon guide catheter (Shuttle-SL [Cook, Bloomington, ID, USA], FlowGate2 [Stryker Neurovascular, Fremont, CA, USA]) was placed in the distal CCA or cervical ICA after femoral artery puncture for anterior circulation approach. Microguidewire and microcatheter were used for navigation, and an intermediate catheter was used if necessary. Thrombectomy with aspiration catheter or retriever stent was performed. In posterior circulation, thrombectomy was performed after the shuttle sheath or guiding catheter was

Table 1. Pial arterial filling score within the symptomatic ischemic territory using single-phase CT angiography (menon pial arterial filling scale*)

\begin{tabular}{l}
\begin{tabular}{l} 
Score \\
\hline 5 \\
4 \\
When compaired with asymptomatic contralateral hemisphere, there is increased or normal prominence and extent of pial vessels \\
When compaired with asymptomatic contralateral hemisphere, there is slightly reduced prominence and extent of pial vessels within \\
the ischemic territory in the symptomatic hemisphere
\end{tabular} \\
$\begin{array}{l}\text { When compaired with asymptomatic contralateral hemisphere, there is moderately reduced prominence and extent of pial vessels } \\
\text { within the ischemic territory in the symptomatic hemisphere }\end{array}$ \\
$\begin{array}{l}\text { When compaired with asymptomatic contralateral hemisphere, there is decreased prominence and extent and regions with no vessels } \\
\text { within the ischemic territory in the symptomatic hemisphere }\end{array}$ \\
\hline When compaired with asymptomatic contralateral hemisphere, there are just a few vessels visiable in the occluded vascular territory
\end{tabular}

*Good pial collateral circulation : score 5 and 4, moderate : score 3 and 2, poor : score 1 and 0. CT : computed tomography 

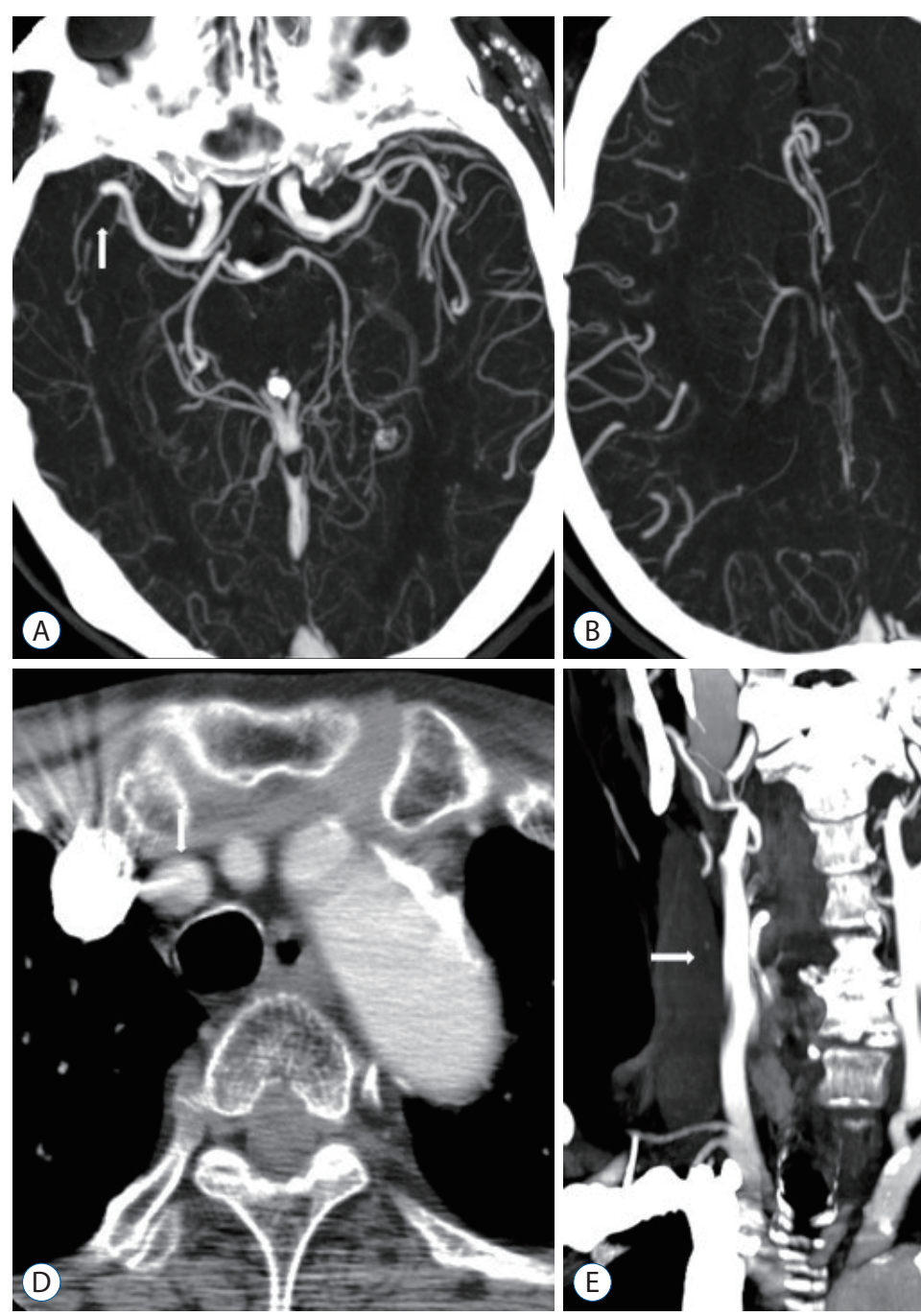

Fig. 2. A 78-year-old woman with a right distal M1 MCA occlusion. OTD 240 minutes, NIHSS 16, DTI 16 minutes, DTP 82 minutes, mTICl 3, 90 days mRS 1. A : MIP intracranial axial image (arrow, right distal M1 MCA occlusion). B and C : Good pial collaterals (score 5). D : Axial image showing the aortic arch (arrow, Brachiocephalic trunk; origin of Brachiocephalic trunk, left CCA, and left subclavian artery). E : MIP extracranial coronal image (arrow, no significant stenosis of right CCA to cervical ICA). F : MIP extracranial sagittal image (arrow, tortuosity of proximal right cervical CCA). MCA : middle cerebral artery, OTD : onset-to-door time, NIHSS : National Institutes of Health Stroke Scale, DTI : door-to-image time, DTP : door-to-puncture time, mTICl : modified thrombolysis in cerebral infarction, mRS : modified Rankin Scale, MIP : maximum intensity projection, CCA : common carotid artery, ICA : internal carotid artery.

placed in proximal VA.

The materials used in the procedure were as follows : Penumbra reperfusion catheter (Penumbra, Alameda, CA, USA), Solitaire stent (Medtronic Neurovascular, Irvine, CA, USA), and a microcatheter (Excelsior SL-10 [Stryker Neurovascular], Prowler Select Plus [Cordis, Dublin, OH, USA] or Rebar [EV3, Irvine, CA, USA]).

The guiding catheter or sheath was continuously flushed with heparinized normal saline. For systemic anticoagulation, a 3000-U bolus of IV heparin was injected at the time of in- troducer sheath insertion by maintaining the infusion rate of $1000-\mathrm{U} / \mathrm{hr}$.

\section{Outcome measures}

The medical records and image data of patients who underwent MT were retrospectively analyzed to investigate demographic and clinical outcomes. The evaluation of vascular recanalization after MT was defined as a modified thrombolysis in cerebral infarction (mTICI) scale : successful recanalization was defined as the value ranging from mTICI $2 b(50-90 \%$ re- 

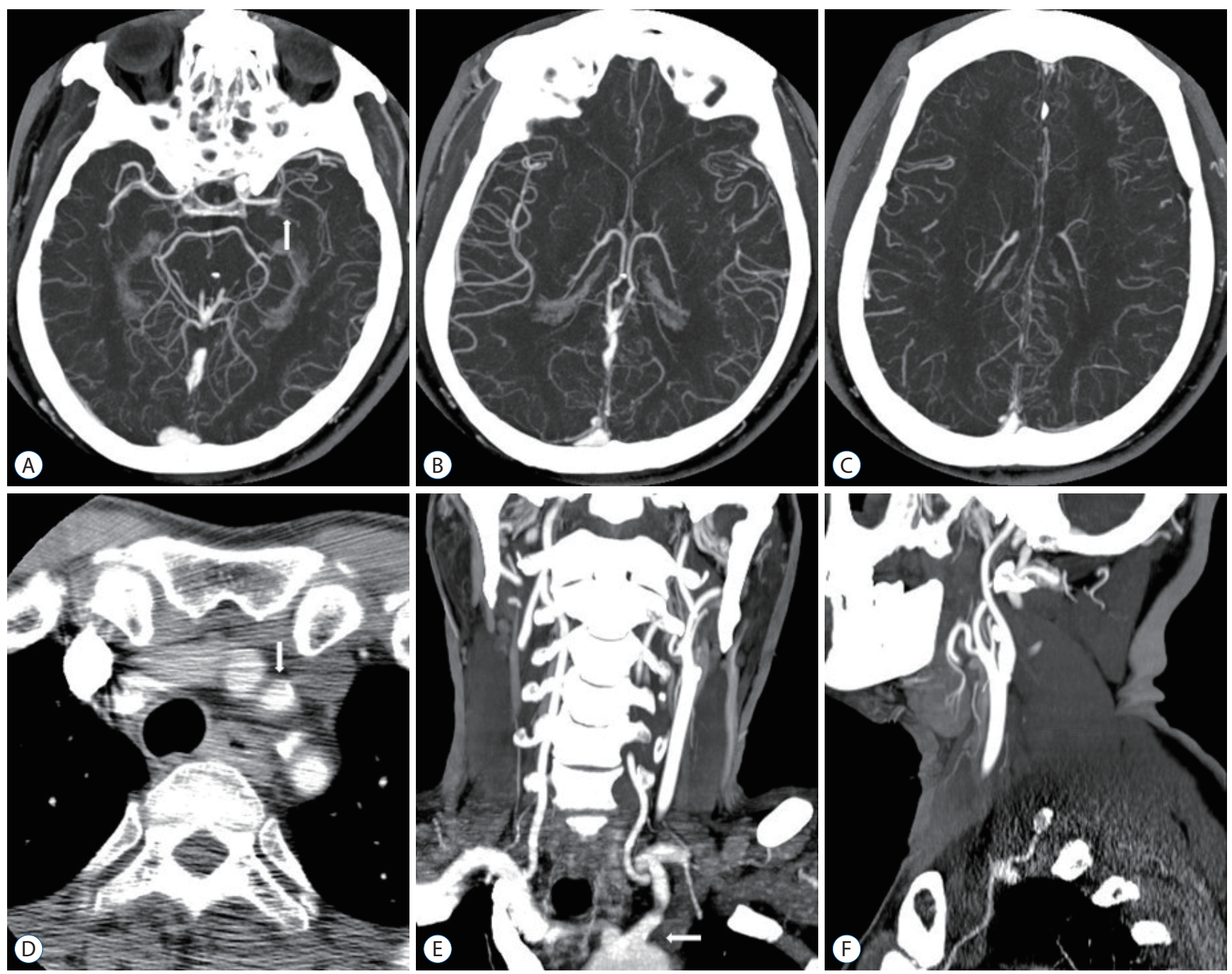

Fig. 3. A 70-year-old man with a left mid M1 MCA occlusion. OTD 22 minutes, NIHSS 12, DTI 6 minutes, DTP 70 minutes, mTICI 2b, 90 days mRS 2. A : MIP intracranial axial image (arrow, left mid M1 MCA occlusion). B and C: Moderate pial collaterals (score 3). D : Axial image showing the aortic arch (arrow, left CCA; origin of Brachiocephalic trunk, left CCA, aberrant left VA, and left subclavian artery). E : MIP extracranial coronal image (arrow, bovine origin of left CCA). F : MIP extracranial sagittal image (no significant stenosis of left CCA to cervical ICA). MCA : middle cerebral artery, OTD : onset-to-door time, NIHSS : National Institutes of Health Stroke Scale, DTI : door-to-image time, DTP : door-to-puncture time, mTICI : modified thrombolysis in cerebral infarction, mRS : modified Rankin Scale, MIP : maximum intensity projection, CCA : common carotid artery, VA : vertebral artery, ICA : internal carotid artery.

perfusion) to 3 (complete reperfusion). NIHSS was assessed at the time of the visit to the ER and at the time of discharge and modified Rankin Scale (mRS) was assessed at after 90 days of the visit. A good functional outcome was defined as a mRS score of 2 or less. Procedure-related symptomatic intracerebral hemorrhage (ICH) including subarachnoid hemorrhage (SAH) was defined as the presence of hemorrhage after MT.

In the CTA group, time to CTA imaging after symptom onset (onset-to-image, door-to-image), time to arterial puncture (onset-to-puncture, door-to-puncture), and reperfusion (on- set-to-reperfusion, door-to-reperfusion) were characterized as time-related outcomes. In the NCCT+MRi group, time to MRI imaging after symptom onset and time to puncture and reperfusion after NCCT imaging and MRI imaging were characterized. Mortality was defined as death within 90 days from the onset of symptoms.

Statistical analysis was performed using SPSS version 25.0 (IBM, Armonk, NY, USA). Demographics, outcomes, and group comparisons were analyzed using the t-test and the chisquared test as appropriate. Statistically significant differences 

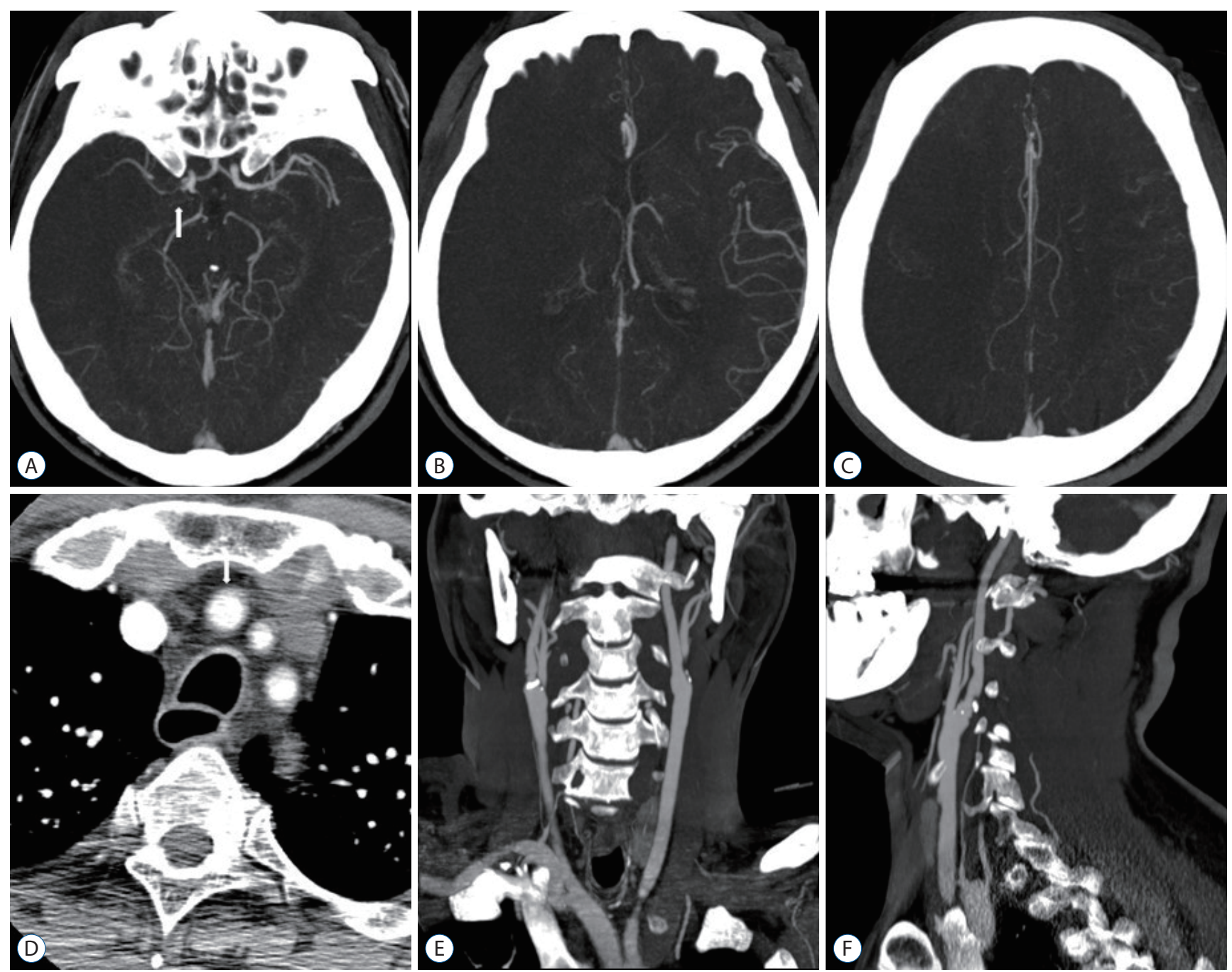

Fig. 4. A 63-year-old man with a right proximal M1 MCA occlusion. OTD 48 minutes, NIHSS 18, DTI 14 mintues, DTP 67 minutes, mTICI 3, 90 days mRS 5. A : MIP intracranial axial image (arrow, right proximal M1 MCA occlusion). B and C: Poor pial collaterals (score 0). D : Axial image showing the aortic arch (arrow, Brachiocephalic trunk; origin of Brachiocephalic trunk, left CCA, and left subclavian artery). E and F : MIP extracranial coronal and sagittal images (no significant stenosis of right CCA to cervical ICA). MCA : middle cerebral artery, OTD : onset-to-door time, NIHSS : National Institutes of Health Stroke Scale, DTI : door-to-image time, DTP : door-to-puncture time, $\mathrm{mTICl}$ : modified thrombolysis in cerebral infarction, mRS : modified Rankin Scale, MIP : maximum intensity projection, CCA : common carotid artery, ICA : internal carotid artery.

were defined as $p<0.05$.

\section{RESULTS}

From April 2015 to March 2019, 157 MT patients met the inclusion criteria : 34 in the CTA group and 33 in the NCCT+MRi group. Univariate analysis of the demographics, risk factor, occlusion site, pial collaterals, and IV r-tPA usage is shown in Table 2. The mean age of the patients was noted as
$71.1 \pm 10.9$ years (mean \pm standard deviation) in the CTA group and $67.0 \pm 11.5$ years in the NCCT+MRi group; $62 \%$ in CTA group and $67 \%$ in NCCT+MRi group were males. Sites of occlusion were as follows : ICA (13/34 in CTA group vs. 12/33 in NCCT+MRi group); MCA (13/34 vs. 16/33); ACA (3/34 vs. 0/33); PCA (1/34 vs. $1 / 33)$; BA (2/34 vs. $4 / 33)$; and VA (2/34 vs. 0/33). IV r-tPA was given to $73 \%$ of patients in the CTA group and $60 \%$ of patients in the NCCT+MRi group.

The time delay comparison between the two groups is shown in Table 3 . We found a significantly shorter delay of 
Table 2. Baseline characteristics, received $r$-tPA in the CTA and NCCT+MRi group

\begin{tabular}{|c|c|c|c|}
\hline & CTA $(n=34)$ & NCCT+MRi $(n=33)$ & $p$-value \\
\hline Age (years) & $71.1 \pm 10.9$ & $67.0 \pm 11.5$ & 0.134 \\
\hline Sex, male & $21(62.8)$ & $22(67)$ & 0.800 \\
\hline \multicolumn{4}{|l|}{ Risk factor } \\
\hline Hypertension & $18(52.9)$ & $16(48.4)$ & \\
\hline Diabetes mellitus & $3(8.8)$ & $2(6.0)$ & \\
\hline Arterial fibrillation & $9(26.4)$ & $4(12.1)$ & \\
\hline Hyperlipidemia & $0(0.0)$ & $1(3.0)$ & \\
\hline Smoking & $10(29.4)$ & $15(45.4)$ & \\
\hline History of stroke & $3(8.8)$ & $1(3.0)$ & \\
\hline Other heart disease & $2(5.8)$ & $1(3.0)$ & \\
\hline \multicolumn{4}{|l|}{ Occlusion site } \\
\hline ICA & $13(38.2)$ & $12(36.4)$ & \\
\hline MCA & $13(38.2)$ & $16(48.5)$ & \\
\hline ACA & $3(8.9)$ & $0(0.0)$ & \\
\hline PCA & $1(2.9)$ & $1(3.0)$ & \\
\hline $\mathrm{BA}$ & $2(5.9)$ & $4(12.1)$ & \\
\hline VA & $2(5.9)$ & $0(0.0)$ & \\
\hline \multicolumn{4}{|l|}{ Pial collaterals } \\
\hline Poor & $11(32.4)$ & & \\
\hline Moderate & $13(38.2)$ & & \\
\hline Good & $10(29.4)$ & & \\
\hline Received r-tPA & $25(73.5)$ & $20(60.6)$ & \\
\hline
\end{tabular}

Values are presented as mean \pm standard deviation or number (\%). r-tPA : recombinant tissue plasminogen activator, CTA : computed tomography angiography, NCCT : non-contrast computed tomography, MRi : magnetic resonance image, ICA : internal carotid artery, MCA : middle cerebral artery, ACA : anterior cerebral artery, PCA : posterior cerebral artery, BA : basilar artery, VA : vertebral artery

door-to-puncture time (mean, $86 \pm 22.1$ vs. $176 \pm 47.5$ minutes; $p<0.01$ ). Also, a significantly shorter total stroke imaging duration was observed in the CTA group (mean, $13 \pm 6.8$ vs. $93 \pm$ 30.8 minutes; $p<0.01$ ). A significantly shorter mean interval from the onset of symptoms to arterial puncture was observed (mean, $195 \pm 128.0$ vs. $314 \pm 157.6$ minutes; $p<0.01$ ). There was no significant difference between the groups from the onset of the symptoms to imaging initiation. Furthermore, there was no significant difference in duration from an arterial puncture to reperfusion time between the two groups. Baseline characteristics, reperfusion result, and clinical outcomes are shown in Table 4.

Reperfusion result of mTICI $\geq 2 \mathrm{~b}$ was $100 \%$ (34/34) in the
Table 3. Comparison of duration (minutes) between the CTA and NCCT+MRi group

\begin{tabular}{|c|c|c|c|}
\hline & CTA $(n=34)$ & $\begin{array}{c}\text { NCCT+MRi } \\
(n=33)\end{array}$ & $p$-value \\
\hline Onset-to-door time & $109.0 \pm 119.1$ & $141.0 \pm 137.2$ & 0.308 \\
\hline Door-to-image time & $13.0 \pm 6.8$ & $93.0 \pm 30.8$ & $0.000^{*}$ \\
\hline Door-to-puncture time & $86.0 \pm 22.1$ & $177.0 \pm 47.5$ & $0.000^{*}$ \\
\hline Door-to-reperfusion time & $122.0 \pm 32.7$ & $211.0 \pm 50.1$ & $0.000^{*}$ \\
\hline Onset-to-image time & $122.0 \pm 119.0$ & $231.0 \pm 144.7$ & $0.001^{*}$ \\
\hline Onset-to-puncture time & $195.0 \pm 128.0$ & $314.0 \pm 157.6$ & $0.001^{*}$ \\
\hline Onset-to-reperfusion time & $231.0 \pm 128.9$ & $332.0 \pm 142.7$ & $0.001^{*}$ \\
\hline
\end{tabular}

Table 4. Reperfusion result and clinical outcomes in the CTA and NCCT+MRi group

\begin{tabular}{lccc}
\hline & CTA $(\mathbf{n}=34)$ & NCCT+MRi $(\mathbf{n}=33)$ & $p$-value \\
\hline Final mTICl & & & \\
$0-2 a$ & $0(0.0)$ & $2(6.1)$ & \\
$2 b$ & $9(26.5)$ & $7(21.2)$ & \\
3 & $25(73.5)$ & $24(72.7)$ & \\
NIHSS & & & \\
Baseline & $18.6 \pm 5.4$ & $15.0 \pm 5.6$ & $0.010^{*}$ \\
Discharge & $7.2 \pm 7.4$ & $8.1 \pm 7.5$ & 0.234 \\
mRS at 90days (0-2) & $23(67.6)$ & $20(60.6)$ & 0.663 \\
\hline
\end{tabular}

Values are presented as mean \pm standard deviation or number (\%). ${ }^{*} p<0.05$. CTA : computed tomography angiography, NCCT : non-contrast computed tomography, MRi : magnetic resonance image, $\mathrm{mTICI}$ : modified thrombolysis in cerebral infarction, NIHSS : National institutes of Health Stroke Scale, mRS : modified Rankin Scale

CTA group and 94\% (31/33) in the NCCT+MRi group, and the result of mTICI 3 was $74 \%$ (25/34) in the CTA group and $73 \%$ (24/33) in the NCCT+MRi group. Baseline NIHSS score was $18.6 \pm 5.4$ vs. $15.0 \pm 5.6$, which improved to $7.2 \pm 7.4$ vs. $8.1 \pm$ 7.5 by the time of discharge. Favorable functional outcomes (mRS score $\leq 2$ at 90 days) was reported as $68 \%(23 / 34)$ in the CTA group and 60\% (20/33) in the NCCT+MRi group, with occurrence of three deaths in the CTA group (cardiac problem 1 and poor pial collaterals 2) and two in the NCCT+MRi group (pulmonary problem 1 and ICH 1). There were three cases of procedure-related complications (ICH 2, SAH 1) in only NCCT+MRi group. Regarding clinical outcomes, although there were significant differences in baseline NIHSS, 
there was no significant difference between the mRS in both the groups at 90 days (Table 4).

\section{DISCUSSION}

Recanalization of acute cerebral artery occlusion is a procedure that significantly improves a patient's quality of life ${ }^{9)}$. In many studies, the main prognostic factors are indicated as early recanalization, good collateral, small core infarct, low NIHSS, and young age ${ }^{12,16)}$. Early recanalization is the most important factor for a good prognosis ${ }^{2,3,16,36)}$. The IV r-tPA within 4.5 hours of stroke is an effective treatment for AIS ${ }^{17}$. However, low recanalization rates are still limited when the occlusion area is in the proximal region and the narrow therapeutic window ${ }^{28)}$. To overcome these limitations, EVT including chemical thrombolysis and various MT were introduced within 6-8 hours of the occurrence of symptoms ${ }^{20)}$.

MT of large intracranial vessels has been mentioned in most of the previous studies ${ }^{43)}$. Currently, MT is a widely used method and many studies have shown good results of early recanalization due to low complications ${ }^{26)}$. Generally, the main instrument of EVT for AIS is stent-retrievers, which shows the efficacy and favorable clinical outcomes in several comparative randomized trials ${ }^{38)}$. Several recent reports have described manual aspiration techniques known as manual aspiration thrombectomy, forced suction thrombectomy, or the a direct aspiration first pass technique techniques, and demonstrated favorable results on recanalization rates, cost efficiency, procedural duration, and functional outcomes ${ }^{19,22,49)}$.
To achieve early recanalization, great efforts have been made to minimize the time to arterial puncture after patients arrival at the hospital. Reducing the time required for preliminary examination for decision making can also be used as an approach to reduce the time for early recanalization ${ }^{41,45)}$. These efforts have been well discussed in several studies on NCCT+CTA, CTP, and MRI ${ }^{3,6,16,21)}$. The five positive trials published in 2015 led to guideline changes in many countries in the process of updating their recommendations for EVT for ischemic stroke ${ }^{7}$ (Table 5). In the present study, we proposed that single-phase CTA including NCCT and MIP images, as a sole examination for decision making prior to the procedure, can significantly reduce door-to-puncture time, which can contribute greatly to early recanalization.

Multimodal MRI shows various useful parameters in determining treatment routes of $\mathrm{AIS}^{24)}$. This technique is especially useful if stroke diagnosis is uncertain (stroke-mimics), stroke onset time is uncertain (un-witnessed daytime strokes or wake-up), or there are clinic-imaging mismatches. However, despite potential benefits of MRI, there are practical problems limiting use of MRI in AIS ${ }^{48}$. One of the major problems is longer scan duration compared to CT imaging. In general, decision-making of thrombolysis based on MRI extended doorto-needle time, but clinical results were more favorable ${ }^{9,50)}$. Thus, use of MRI in treating acute stroke is clinically practical and feasible ${ }^{23,35)}$. Conversely, considering the "time is brain" in the hyperacute phase of ischemic stroke, minimizing evaluation time required to determine treatment could expand the benefits of thrombolysis ${ }^{24)}$.

In our study, 30 of 157 patients who performed MT tested

Table 5. Characteristics of endovascular thrombectomy trials ${ }^{7)}$

\begin{tabular}{|c|c|c|c|c|c|c|c|}
\hline Trial & Number & $\begin{array}{l}\text { Onset to puncture } \\
\text { limit (hours) }\end{array}$ & $\begin{array}{l}\text { NIHSS } \\
\text { limits }\end{array}$ & $\begin{array}{l}\text { Median } \\
\text { NIHSS }\end{array}$ & Pre-MT imaging & $\begin{array}{l}\text { Onset-to-puncture } \\
\text { time (minutes) }\end{array}$ & $\begin{array}{c}\text { Successful } \\
\text { recanalization } \\
(\mathrm{mTICl} 2 \mathrm{~b} / 3, \%)\end{array}$ \\
\hline IMS 3 & 653 & 6 & $\geq 10$ & 17 & NCCT & 208 & 41 \\
\hline MR CLEAN & 500 & 6 & $\geq 2$ & 18 & $\mathrm{CT}+\mathrm{CTA}$ & 260 & 59 \\
\hline ESCAPE & 316 & 12 & $\geq 6$ & 17 & $\mathrm{CT}+\mathrm{CTA}( \pm \mathrm{CTP})$ & 200 & 76 \\
\hline EXTEND-IA & 70 & 6 & No limits & 15 & $\mathrm{CT}+\mathrm{CTA}+\mathrm{CTP}$ & 210 & 86 \\
\hline SWIFT PRIME & 196 & 6 & $\geq 8$ & 14 & $\mathrm{CT}+\mathrm{CTA}( \pm \mathrm{CTP}$ or $\mathrm{MRI})$ & 224 & 88 \\
\hline REVASCAT & 206 & 8 & $\geq 6$ & 17 & $\mathrm{CT}+\mathrm{CTA}( \pm \mathrm{CTP})$ & 269 & 66 \\
\hline
\end{tabular}

NIHSS : National Institutes of Health Stroke Scale, MT : mechanical thrombectomy, mTICl : modified thrombolysis in cerebral infarction, NCCT : noncontrast computed tomography, CT : computed tomography, CTA : computed tomography angiography, CTP : computed tomography perfusion, MRI : magnetic resonance image 
additional MRI after CTA. Additional MRI imaging was required in case decision-making for MT was not possible with CTA alone; if it was not possible to confirm an occlusion site that matched symptoms (high NIHSS score but not sure of occlusion, patients with LVO but low NIHSS score), in cases the onset time was unclear (symptoms onset within 12 hours to 24 hours, wake-up, un-witnessed daytime strokes). If the occlusion site could be confirmed (old lesion or new lesion), and it was similar to symptoms caused by internal disease, it was known there was a stroke (stroke-mimics). If sufficient evidence was not created for determination by CTA alone, further MRI imaging was required, and it was found that MRI was an effective tool for decision-making of $\mathrm{MT}^{24)}$ (Fig. 5).

CTA has many advantages over NCCT, such as high sensitivity to LVO detection, visualization of carotid vessels, and tortuosity of aortic arch as part of stroke workup and thrombectomy planning, especially under conditions of difficult vessel anatomy (including femoral artery or radial artery approach) to select an appropriate approach to vessel access ${ }^{29)}$. Structural variation in the aortic arch is rarely reported and should be considered in terms of the approach in the procedure $^{10)}$.

Data from previous trials reveals that clinical outcome improves if patients have a salvageable brain at presentation and undergo early recanalization ${ }^{5,33,40)}$. Risk of poor clinical outcomes can increase by approximately 14\% each time treatment is delayed every 30 minutes $^{31}$. So, the ideal imaging tool should detect the salvageable brain quickly and reliably ${ }^{32}$. Current imaging techniques include non-enhanced CT, single-phase or multiphage CTA, CTP, and MRI imaging. Singlephase CTA and Multiphase CTA provide clinicians with information on degree and extent of pial arterial filling throughout the brain. Multiphase CTA provides clinicians with multiple time-resolution images, providing a more subtle
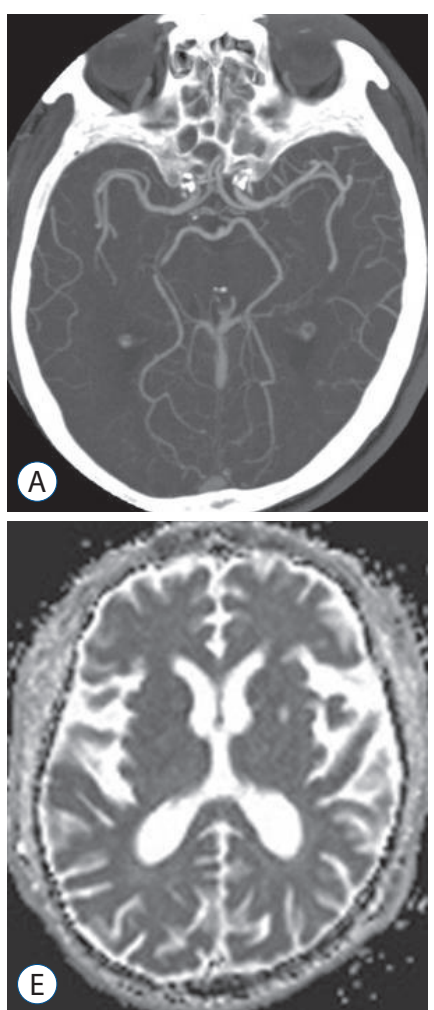
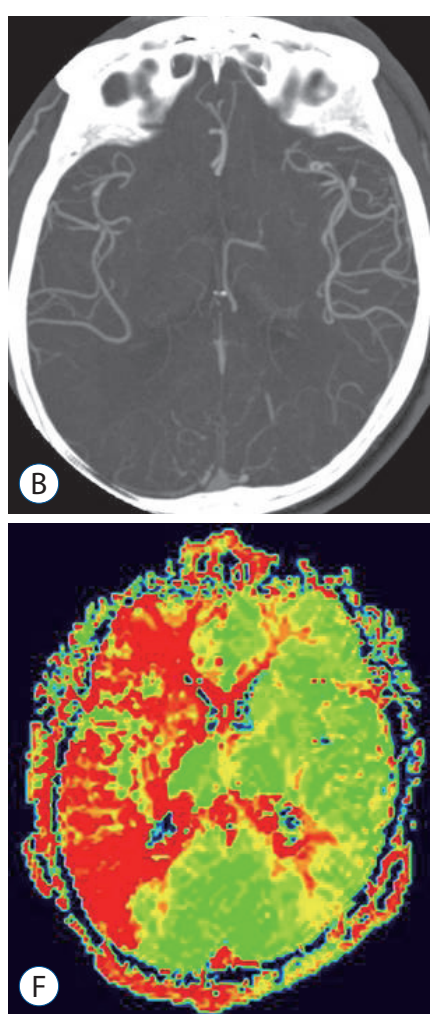
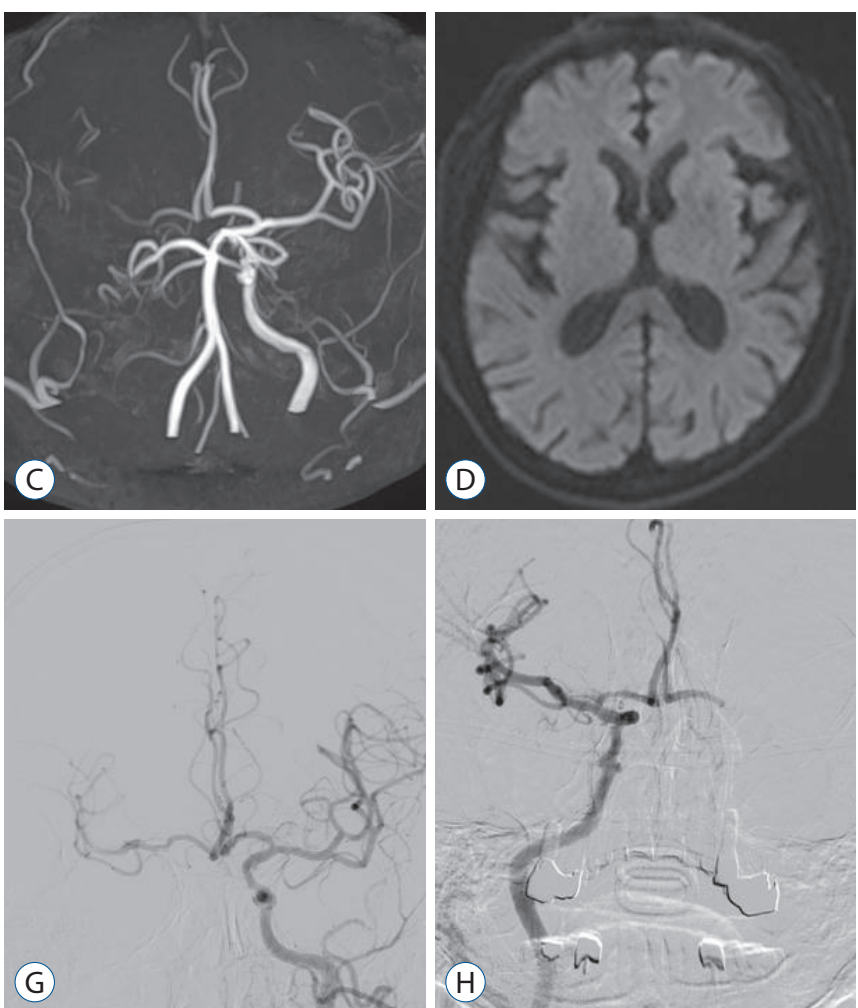

Fig. 5. A 80-year-old man with a right proximal cervical ICA occlusion. OTD 304 minutes, NIHSS 21, DTI 78 minutes, DTP 164 minutes, mTICI 3, 90 days mRS 0. A and B : MIP intracranial axial image (no occlusion, good pial collaterals). C : MRA image (occlusion of ICA and weak flow of MCA). D-F : MRI images (perfusion-diffusion mismatch). G : Left ICA angiography (pre-MT, irrigation of contralateral ACA and MCA). H : Right ICA angiography (post-MT, successful recanalization, mTICI 3). ICA : internal carotid artery, OTD : onset-to-door time, NIHSS : National Institutes of Health Stroke Scale, DTI : door-toimage time, DTP : door-to-puncture time, $\mathrm{mTICl}$ : modified thrombolysis in cerebral infarction, mRS : modified Rankin Scale, MIP : maximum intensity projection, MRA : magnetic resonance angiography, MCA : middle cerebral artery, MRI : magnetic resonance image, MT : mechanical thrombectomy, ACA : anterior cerebral artery. 
assessment of pial arterial filling in the normal brain and ischemic brain ${ }^{32}$. Single-phase CTA has a limitation on lack of temporal resolution; so, this modality leads to risk of mislabeling pial arterial filling when compared with multiphase $\mathrm{CTA}^{14,34)}$.

Serum creatinine levels are generally evaluated before performing CTA due to potential risk of contrast-induced acute renal damage. However, many studies have shown that incidence $(2 \%$ to $4 \%$ ) of contrast-induced nephropathy ( $>25 \%$ increase in creatinine) in AIS patients who have undergone emergency CT scanning or emergency EVT is low and is not significantly different from other conditions ${ }^{4,47)}$. There is controversy as to if it is justifiable to perform CTA (or DSA) without baseline serum creatinine in hyperacute ischemic stroke ${ }^{4)}$. However, considering the rapid neuronal death rate (2 million per minute) and patients' outcomes, harm caused by delayed treatment in AIS patients is greater than the benefit of preventing low incidence, and mainly reversible, renal damage. So, in cases of possible stroke with no history of clinical evidence of renal impairment or severe dehydration, doctors do not need to wait for laboratory results before $\mathrm{CTA}^{8,25)}$.

CTA after NCCT before thrombectomy to confirm LVO is widely performed in many institutions based on protocols used for LVO stroke thrombectomy in large clinical trials recently announced ${ }^{44)}$. All the positive trials required LVO evidence using non-invasive angiography (CTA). Especially when visualized using thin-slice NCCT, the apparent high density artery appears to have high sensitivity and speciality to acute occlusive thrombus ${ }^{42)}$. However, the CTA for occlusion is considered a standard procedure and should be extended from the aorta arch to allow evaluation of proximal vascular approaches to plan an endovascular approach ${ }^{27,39)}$.

Some of the institutions have attempted to selectively conduct CTA in cases with severe stroke involving frequent LVO. However, patients with mild clinical symptoms still have a significant incidence of LVO (approximately 10\% in those with NIHSS <6) and these patients are at a risk of further clinical deterioration ${ }^{11)}$. The routine imaging method using both NCCT and CTA is simple, and uniform workflow is likely to result in faster and higher quality acquisition due to the familiarity of radiologists ${ }^{7}$.

In the present study, it was observed that avoiding extra vascular images (selective CTA after NCCT or MRI after NCCT) in patients with CTA (including NCCT and MIP) images showing occlusion sites significantly reduced the door-topuncture time. The observed improvement in time in our patient cohort was approximately 90 minutes. Reduced reperfusion time is related to improvement in functional outcomes and by optimizing the time delay before arterial puncture, improvement in time delay could be achieved ${ }^{5,31,33,40)}$. Although CTA group did not produce better clinical outcomes than NCCT+MRi group in our study, we achieved as favorable functional outcomes as the NCCT+MRi group. As with recent attempts, it was possible to conclude that reperfusion time is an effort to reduce evaluation time and that it is valuable to consider it as the only preliminary examination for MT.

Our study has several limitations. The data was collected retrospectively without randomized allocation, and the sample size was relatively small and associated with selection bias. The decision of EVT or extra imaging was made by the neurointerventionist on-call doctor, although we had an indication of MT. The usage of variable devices, like the size of the sheath, guiding catheter, intermediate catheter, micro catheter, guidewire, aspiration catheter or stent, and size of materials were decided based on the cases. Furthermore, various limitations, such as prognosis change according to r-tPA usage status, selection of patients with treatment in the NCCT+MRi group, more objective NIHSS/mRS assessment, subdivided assessment of clinical outcomes, and determination of initial imaging selection by on-call doctor, should be considered thoroughly. Also, several clinical and radiological factors which could affect the outcomes should be considered. A larger prospective trial is needed to further confirm our findings.

\section{CONCLUSION}

A single-phase CTA including NCCT and MIP images was performed as a single preliminary examination to reduce the time from the arrival of the patient to the hospital to the start of the procedure, which not only reduced the time for the initiation of the procedure but also led to good results in terms of prognosis. So, it is appropriate to conclude that the proposed method holds significant value as the only preliminary examination for decision-making of MT in patients with LVO stroke. Although our study did not achieve better clinical outcomes compared to the NCCT+MRi group, we produced 
good results like the NCCT+MRi group and confirmed that it was a way to significantly reduce reperfusion time.

\section{CONFLICTS OF INTEREST}

No potential conflict of interest relevant to this article was reported.

\section{INFORMED CONSENT}

Informed consent was obtained from all individual participants included in this study.

\section{AUTHOR CONTRIBUTIONS}

\author{
Conceptualization : MSK, GSK \\ Data curation : MSK, GSK \\ Formal analysis : MSK, GSK \\ Funding acquisition : MSK, GSK \\ Methodology : MSK, GSK \\ Project administration : MSK, GSK \\ Visualization : MSK, GSK \\ Writing - original draft : MSK, GSK \\ Writing - review \& editing : MSK, GSK
}

\section{ORCID}

Myeong Soo Kim https:/orcid.org/0000-0003-4542-2912

Gi Sung Kim https://orcid.org/0000-0002-9040-4054

\section{References}

1. Aghaebrahim A, Streib C, Rangaraju S, Kenmuir CL, Giurgiutiu DV, Horev $A$, et al. : Streamlining door to recanalization processes in endovascular stroke therapy. J Neurointerv Surg 9 : 340-345, 2017

2. Akins PT, Amar AP, Pakbaz RS, Fields JD; SWIFT Investigators : Complications of endovascular treatment for acute stroke in the SWIFT trial with solitaire and Merci devices. AJNR Am J Neuroradiol 35 : 524528, 2014

3. Berkhemer OA, Fransen PS, Beumer $D$, van den Berg LA, Lingsma HF,
Yoo AJ, et al. : A randomized trial of intraarterial treatment for acute ischemic stroke. N Engl J Med 372 : 11-20, 2015

4. Brinjikji W, Demchuk AM, Murad MH, Rabinstein AA, McDonald RJ, McDonald JS, et al. : Neurons over nephrons: systematic review and metaanalysis of contrast-induced nephropathy in patients with acute stroke. Stroke 48 : 1862-1868, 2017

5. Broderick JP, Schroth G : What the SWIFT and TREVO II trials tell us about the role of endovascular therapy for acute stroke. Stroke 44 : 1761-1764, 2013

6. Campbell BC, Mitchell PJ, Kleinig TJ, Dewey HM, Churilov L, Yassi N, et al. : Endovascular therapy for ischemic stroke with perfusion-imaging selection. N Engl J Med 372 : 1009-1018, 2015

7. Campbell BCV, Donnan GA, Mitchell PJ, Davis SM : Endovascular thrombectomy for stroke: current best practice and future goals. Stroke Vasc Neurol 1 : 16-22, 2016

8. Casaubon LK, Boulanger JM, Blacquiere D, Boucher S, Brown K, Goddard T, et al. : Canadian stroke best practice recommendations: hyperacute stroke care guidelines, update 2015. Int J Stroke 10 : 924-940, 2015

9. Chalela JA, Kidwell CS, Nentwich LM, Luby M, Butman JA, Demchuk $A M$, et al. : Magnetic resonance imaging and computed tomography in emergency assessment of patients with suspected acute stroke: a prospective comparison. Lancet 369 : 293-298, 2007

10. Choi Y, Chung SB, Kim MS : Prevalence and anatomy of aberrant right subclavian artery evaluated by computed tomographic angiography at a single institusion in Korea. J Korean Neurosurg Soc 62 : 175-182, 2019

11. Coutts SB, Modi J, Patel SK, Demchuk AM, Goyal M, Hill MD, et al. : CT/ $\mathrm{CT}$ angiography and MRI findings predict recurrent stroke after transient ischemic attack and minor stroke: results of the prospective CATCH study. Stroke 43 : 1013-1017, 2012

12. Danière F, Lobotesis K, Machi P, Eker O, Mourand I, Riquelme C, et al. : Patient selection for stroke endovascular therapy--DWI-ASPECTS thresholds should vary among age groups: insights from the RECOST study. AJNR Am J Neuroradiol 36 : 32-39, 2015

13. de Weerd L, Luijckx GJ, Groenier KH, van der Meer K : Quality of life of elderly ischaemic stroke patients one year after thrombolytic therapy. A comparison between patients with and without thrombolytic therapy. BMC Neurol $12: 61,2012$

14. Frölich AM, Wolff SL, Psychogios MN, Klotz E, Schramm R, Wasser K, et al. : Time-resolved assessment of collateral flow using 4D CT angiography in large-vessel occlusion stroke. Eur Radiol 24 : 390-396, 2014

15. García-Tornel A, Carvalho V, Boned S, Flores A, Rodríguez-Luna D, Pagola J, et al. : Improving the evaluation of collateral circulation by multiphase computed tomography angiography in acute stroke patients treated with endovascular reperfusion therapies. Interv Neurol 5 : 209-217, 2016

16. Goyal M, Demchuk AM, Menon BK, Eesa M, Rempel JL, Thornton J, et al. : Randomized assessment of rapid endovascular treatment of ischemic stroke. N Engl J Med 372 : 1019-1030, 2015

17. Hacke W, Kaste M, Bluhmki E, Brozman M, Dávalos A, Guidetti D, et al. : 
Thrombolysis with alteplase 3 to 4.5 hours after acute ischemic stroke. N Engl J Med 359 : 1317-1329, 2008

18. IMS II Trial Investigators : The interventional management of stroke (IMS) II study. Stroke 38 : 2127-2135, 2007

19. Jankowitz B, Aghaebrahim A, Zirra A, Spataru O, Zaidi S, Jumaa M, et al. : Manual aspiration thrombectomy: adjunctive endovascular recanalization technique in acute stroke interventions. Stroke 43 : 1408-1411, 2012

20. Jauch EC, Saver JL, Adams HP Jr, Bruno A, Connors JJ, Demaerschalk BM, et al. : Guidelines for the early management of patients with acute ischemic stroke: a guideline for healthcare professionals from the American Heart Association/American Stroke Association. Stroke 44 : 870947, 2013

21. Jovin TG, Chamorro A, Cobo E, de Miquel MA, Molina CA, Rovira A, et al. : Thrombectomy within 8 hours after symptom onset in ischemic stroke. N Engl J Med 372 : 2296-2306, 2015

22. Kang DH, Hwang YH, Kim YS, Park J, Kwon O, Jung C : Direct thrombus retrieval using the reperfusion catheter of the penumbra system: forcedsuction thrombectomy in acute ischemic stroke. AJNR Am J Neuroradiol 32 : 283-287, 2011

23. Kang DW, Chalela JA, Dunn W, Warach S; NIH-Suburban Stroke Center Investigators : MRI screening before standard tissue plasminogen activator therapy is feasible and safe. Stroke 36 : 1939-1943, 2005

24. Kim BJ, Kang HG, Kim HJ, Ahn SH, Kim NY, Warach S, et al. : Magnetic resonance imaging in acute ischemic stroke treatment. J Storke 16 : 131-145, 2014

25. Kim DH, Kim B, Jung C, Nam HS, Lee JS, Kim JW, et al. : Consensus statements by Korean Society of Interventional Neuroradiology and Korean Stroke Society: hyperacute endovascular treatment workflow to reduce door-to-reperfusion time. J Korean Med Sci 33 : e143, 2018

26. Kim TK, Rhim JK, Lee CJ, Oh SH, Chung BS : The limitations of thrombectomy with Solitaire ${ }^{\mathrm{TM}} \mathrm{AB}$ as first-line treatment in acute ischemic stroke: a single center experience. J Cerebrovasc Endovasc Neurosurg 14 : 203-209, 2012

27. Lee JS, Demchuk AM : Choosing a hyperacute stroke imaging protocol for proper patient selection and time efficient endovascular treatment: lessons from recent trials. J Stroke 17 : 221-228, 2015

28. Lee KY, Han SW, Kim SH, Nam HS, Ahn SW, Kim DJ, et al. : Early recanalization after intravenous administration of recombinant tissue plasminogen activator as assessed by pre- and post-thrombolytic angiography in acute ischemic stroke patients. Stroke 38 : 192-193, 2007

29. Lev MH, Farkas J, Rodriguez VR, Schwamm LH, Hunter GJ, Putman CM, et al. : CT angiography in the rapid triage of patients with hyperacute stroke to intraarterial thrombolysis: accuracy in the detection of large vessel thrombus. J Comput Assist Tomogr 25 : 520-528, 2001

30. Mehta BP, Leslie-Mazwi TM, Chandra RV, Bell DL, Sun CH, Hirsch JA, et al. : Reducing door-to-puncture times for intra-arterial stroke therapy: a pilot quality improvement project. J Am Heart Assoc 3 : e000963, 2014

31. Menon BK, Almekhlafi MA, Pereira VM, Gralla J, Bonafe A, Chapot R, et al. : Optimal workflow and process-based performance measures for endovascular therapy in acute ischemic stroke: analysis of the Solitaire FR thrombectomy for acute revascularization study. Stroke 45 : 20242029, 2014

32. Menon BK, d'Esterre CD, Qazi EM, Almekhlafi M, Hahn L, Demchuk AM, et al. : Multiphase CT angiography: a new tool for the imaging triage of patients with acute ischemic stroke. Radiology 275 : 510-520, 2015

33. Menon BK, Goyal $M$ : Endovascular therapy in acute ischemic stroke: where we are, the challenges we face and what the future holds. Expert Rev Cardiovasc Ther 9 : 473-484, 2011

34. Menon BK, O'Brien B, Bivard A, Spratt NJ, Demchuk AM, Miteff F, et al. : Assessment of leptomeningeal collaterals using dynamic CT angiography in patients with acute ischemic stroke. J Cereb Blood Flow Metab $33:$ 365-371, 2013

35. Merino JG, Warach S: Imaging of acute stroke. Nat Rev Neurol 6 : 560-571, 2010

36. Molina CA, Chamorro A, Rovira À, de Miquel A, Serena J, Roman LS, et al. : REVASCAT: a randomized trial of revascularization with SOLITAIRE FR device vs. best medical therapy in the treatment of acute stroke due to anterior circulation large vessel occlusion presenting within eighthours of symptom onset. Int J Stroke 10 : 619-626, 2015

37. Nogueira RG, Lutseg HL, Gupta R, Jovin TG, Albers GW, Walker GA, et al. : Trevo versus Merci retrievers for thrombectomy revascularisation of large vessel occlusions in acute ischaemic stroke (TREVO 2): a randomised trial. Lancet 380 : 1231-1240, 2012

38. Pereira VM, Gralla J, Davalos A, Bonafé A, Castaño C, Chapot $R$, et al. : Prospective, multicenter, single-arm study of mechanical thrombectomy using Solitaire Flow Restoration in acute ischemic stroke. Stroke 44 : 2802-2807, 2013

39. Powers WJ, Derdeyn CP, Biller J, Coffey CS, Hoh BL, Jauch EC, et al. : 2015 American Heart Association/American Stroke Association focused update of the 2013 guidelines for the early management of patients with acute ischemic stroke regarding endovascular treatment: a guideline for healthcare professionals from the American Heart Association/American Stroke Association. Stroke 46 : 3020-3035, 2015

40. Rha JH, Saver JL : The impact of recanalization on ischemic stroke outcome: a metaanalysis. Stroke 38 : 967-973, 2007

41. Ribo M, Molina CA, Cobo E, Cerdà N, Tomasello A, Quesada H, et al. : Association between time to reperfusion and outcome is primarily driven by the time from imaging to reperfusion. Stroke 47 : 999-1004, 2016

42. Riedel CH, Zoubie J, Ulmer S, Gierthmuehlen J, Jansen 0 : Thin-slice reconstructions of nonenhanced $\mathrm{CT}$ images allow for detection of thrombus in acute stroke. Stroke 43 : 2319-2323, 2012

43. Roth C, Papanagiotou P, Behnke S, Walter S, Haass A, Becker C, et al. : Stent-assisted mechanical recanalization for treatment of acute intracerebral artery occlusions. Stroke 41 : 2559-2567, 2010

44. Saver JL, Goyal M, Bonafe A, Diener HC, Levy El, Pereira VM, et al. : Stent-retriever thrombectomy after intravenous t-PA vs. t-PA alone in stroke. N Engl J Med 372 : 2285-2295, 2015

45. Saver JL, Goyal M, van der Lugt A, Menon BK, Majoie CB, Dippel DW, et al. : Time to treatment with endovascular thrombectomy and outcomes from ischemic stroke: a meta-analysis. JAMA 316 : 1279-1288, 2016 
46. Saver JL, Jahan R, Levy El, Jovin TG, Baxter B, Nogueira RG, et al. : Solitaire flow restoration device versus the Merci Retriever in patients with acute ischaemic stroke (SWIFT): a randomised, parallel-group, noninferiority trial. Lancet 380 : 1241-1249, 2012

47. Sharma J, Nanda A, Jung RS, Mehta S, Pooria J, Hsu DP : Risk of contrast-induced nephropathy in patients undergoing endovascular treatment of acute ischemic stroke. J Neurointerv Surg 5 : 543-545, 2013

48. Singer OC, Sitzer M, du Mesnil de Rochemont R, Neumann-Haefelin T : Practical limitations of acute stroke MRI due to patient-related problems.

\section{Neurology 62 : 1848-1849, 2004}

49. Turk AS, Spiotta A, Frei D, Mocco J, Baxter B, Fiorella D, et al. : Initial clinical experience with the ADAPT technique: a direct aspiration first pass technique for stroke thrombectomy. J Neurointerv Surg 6 : 231237, 2014

50. Yoo SH, Kwon SU, Lee DH, Kim SJ, Kim JS, Kang DW : Comparison between MRI screening and CT-plus-MRI screening for thrombolysis within 3 h of ischemic stroke. J Neurol Sci 294 : 119-123, 2010 